\title{
Programming in the recently-updated pre-university maths curriculum - Further Pure Mathematics with Technology (FPT)
}

Stephen Lee, Mathematics in Education and Industry, Trowbridge, UK. Email:

Stephen.lee@mei.org.uk.

Tom Button, Mathematics in Education and Industry, Trowbridge, UK. Email:

Tom.button@mei.org.uk.

Keywords: A level, curriculum, programming, pure, technology.

\section{Background}

In the article 'Moving with the times - a new A level Further Mathematics Unit: Further Pure Mathematics with Technology (FPT)', Lee and Button (2013) wrote about the optional A level Further Mathematics unit that featured programming. Since then both GCSE and A level Mathematics/Further Mathematics have been revised (see Baldwin and Lee, 2017, and Glaister, 2017).

A level Mathematics and Further Mathematics changed for first teaching in academic year 2017/18 and thus, after completing these maths qualifications over two years, those students would have predominantly entered university in the current academic year 2019/20 (Note: a small number may have completed the new courses in one year and entered university in 2018/19). A level Mathematics is still the most popular of all subjects and in summer 2019 there were 91,895 entries in the UK; 14,527 studied A level Further Mathematics (Joint Council for Qualifications data). However, this was a decrease of $5.9 \%$ and $10.1 \%$ respectively from 2018 .

The major change is that $A$ levels are no longer modular courses; students are assessed by end of course examinations. The content of A level Mathematics is completely prescribed; however, the content of $A$ level Further Mathematics still retains some element of choice over some of the content. The Further Pure with Technology unit has been updated and is still available as an optional component for Further Mathematics students and in this short update we will address its role and how it can support pre-university students in developing their mathematical programming skills.

\section{Aim and philosophy of FPT}

The aim and philosophy of FPT is to support students in developing their skills in using technology for pure mathematics. There are three topics studied: investigation of curves, differential equations and number theory. In investigation of curves students use a graph-plotter and Computer Algebra System (CAS); in differential equations they use a graph-plotter, CAS and a spreadsheet; in number theory they use a programming language (they are required to use Python in this course). The common approach in all of these topics is that students utilise the power of technology to find or generate mathematics that would be time-consuming or difficult to create by 'hand', and once they have done so they use their mathematical skills to explain the results obtained.

For example, in the number theory topic students work on questions such as that in Figure 1. 
(i) Write a program to solve the congruence $x^{2} \equiv 0(\bmod n)$.

(ii) Use your program to find the solutions to:

(a) $x^{2} \equiv 0(\bmod 101)$

(b) $x^{2} \equiv 0(\bmod 112)$

(c) $x^{2} \equiv 0(\bmod 1009)$

(iii) Show that if $p$ is prime then $x=0$ is the only solution to $x^{2} \equiv 0(\bmod p)$.

Figure 1. Example number theory question from FPT.

Technology is used here to systematically work through a large number of cases but, once the results have been obtained, students are expected to give a rigorous mathematical reason for the statement in part (iii). In FPT the emphasis is on students having an understanding of the mathematics and for the technology to support them in this; the questions are intended to model the practice of searching a large number of cases but then being able to justify and explain any results.

In FPT all the programs are written in Python. However, this is not a programming course; the only expectation of the students is that they can use the mathematical functions built-in to Python with the 'for' and 'if' commands. They are then expected to be able to write short programs to perform exhaustive (or brute-force) searches. Number theory is an ideal topic for this as many of the problems relate to positive integers which are straightforward to generate with the for command. This is also a valuable topic as students only real experience of number theory otherwise is a brief introduction to the concept of prime numbers lower down the school. Many A level Further Mathematics students will be considering progressing to a degree in Mathematics. Studying number theory in A level Further Mathematics provides an opportunity for them to meet a topic that more resembles undergraduate mathematics than much of A level Mathematics.

An example question from the specimen paper is shown in Figure 2. This demonstrates the standard students are expected to reach by the time they take their examinations. The full specimen paper and other related information on the FPT component of Further Mathematics can be found on the MEl website: www.mei.org.uk/fpt

\section{FPT in the new ' 2017 ' curriculum}

As stated in section 1, for the new 2017 specifications A level Further Mathematics has some compulsory content. More specifically, $50 \%$ is a prescribed 'pure maths core'. For the remaining $50 \%$ of the content, different options are available. These options vary between awarding organisations (AQA/Edexcel/OCR/OCR (MEI)) and may include mechanics, statistics, discrete/decision maths and additional pure maths. For the OCR (MEI) specification FPT makes up $162 / 3 \%$ of $A$ Level Further Mathematics content.

OCR (MEI) A level Further Mathematics also allows students to take additional options, with the best scores contributing to their A level grade, so FPT could be offered to students as a useful additional option, without committing to it being part of their overall mark.

One of the key points to emphasise is that even within the parameters required for devising a specification from 2017, MEI was able to develop an innovative curriculum involving aspects like FPT. An equivalent to FPT is not found in any of the other specifications. 
(i) $(A)$ Create a program to find all the solutions to $x^{2} \equiv-1(\bmod p)$ where $0 \leq x<p$.

Write out your program in full in the Printed Answer Booklet.

(B) Use the program to find the solutions to $x^{2} \equiv-1(\bmod p)$ for the primes

- $p=809$,

- $p=811$ and

- $p=444001$.

(ii) State Wilson's Theorem.

(iii) The following argument shows that $(4 k) ! \equiv((2 k) !)^{2}(\bmod p)$ for the case $p=4 k+1$.

$$
\begin{aligned}
(4 k) ! & \equiv 1 \times 2 \times 3 \times \ldots \times(2 k-1) \times 2 k \times(2 k+1) \times(2 k+2) \times \ldots \times(4 k-1) \times 4 k(\bmod p) \\
& \equiv 1 \times 2 \times 3 \times \ldots \times(2 k-1) \times 2 k \times(-2 k) \times(-(2 k-1)) \times \ldots \times(-2) \times(-1)(\bmod p) \\
& \equiv((2 k) !)^{2}(\bmod p)
\end{aligned}
$$

(A) Explain why $(2 k+2)$ can be written as $(-(2 k-1))$ in line (2).

(B) Explain how line (3) has been obtained.

(C) Explain why, if $p$ is a prime of the form $p=4 k+1$, then $x^{2} \equiv-1(\bmod p)$ will have at least one solution.

(D) Hence find a solution of $x^{2} \equiv-1(\bmod 29)$.

Figure 2. Example question from the FPT specimen paper.

The continuation of having an FPT component, when the new curriculum changed in 2017, also aligns with several of the recommendations of the Smith review into post-16 mathematics (Department for Education, 2017). Smith highlighted use of technology as a key area to maintain pace with the changing world and for teaching and the wider workforce. MEl will continue to innovate in mathematics education to support education and industry in this area.

\section{References}

Baldwin, C. and Lee, S., 2017. Exploring the new AS and A levels in Mathematics and Further Mathematics. Mathematics Today, 53(4), pp.156-158.

Department for Education, 2017. Report of Professor Sir Adrian Smith's review of post-16 mathematics. Available at: https://www.gov.uk/government/publications/smith-review-of-post-16maths-report-andgovernment-response [Accessed 20 February 2020].

Glaister, P., 2017. AS and A levels in Mathematics and Further Mathematics are changing - are you ready? MSOR Connections, 15(7), pp.14-27. https://doi.org/10.21100/msor.v15i3.508.

Lee, S. and Button, T., 2013. Moving with the Times - A New A level Further Mathematics Unit: Further Pure Mathematics with Technology (FPT). MSOR Connections, 13(2), pp.10-11. 